\title{
Agent-based Identification and Control of Voltage Emergency Situations
}

ISSN 1751-8644 doi: 0000000000 www.ietdl.org

\author{
Lena Robitzky ${ }^{1 *}$, Tilman Weckesser ${ }^{2}$, Ulf Häger ${ }^{1}$, Christian Rehtanz ${ }^{1}$, Thierry Van Cutsem ${ }^{3}$ \\ ${ }^{1}$ Institute of Energy Systems, Energy Efficiency and Energy Economics, TU Dortmund University, Emil-Figge Str. 70, 44227 Dortmund, Germany \\ ${ }^{2}$ Department of Electrical Engineering and Computer Science, University of Liège, Sart Tilman B37, 4000 Liège, Belgium \\ ${ }^{3}$ Fund for Scientific Research (FNRS) at the University of Liège, Liège, Belgium \\ *E-mail: lena.robitzky@tu-dortmund.de
}

\begin{abstract}
Changing dynamics of power systems caused by the migration from conventional to distributed energy sources increase the risk of blackouts due to voltage instability, especially in case of unforeseen network conditions (e.g., (N-k)-cases). To enable a both secure and efficient power supply, novel monitoring and emergency control systems for the identification of voltage emergency situations as well as the execution of control actions are required that react reliably in due time and adaptively in case of changing network situations. This paper presents a distributed agent-based approach to counteract voltage instability that is based solely on local measurements and limited inter-agent communication. Distributed agents located at substations in the (sub-)transmission network monitor distribution and transmission voltages as well as load tap changer positions and are able to autonomously curtail load in case system stability is endangered. The applicability of the approach is demonstrated in a co-simulation environment interfacing the multi-agent system with a dynamic power system simulation. The presented approach allows for an early detection of voltage instability as well as a coordinated execution of available control actions.
\end{abstract}

\section{Introduction}

The large-scale integration of renewable energy sources and the related decommissioning of conventional power plants change the complexity and dynamics of future power grids. Especially in case of $(\mathrm{N}-\mathrm{k})$ situations, the risk of regional or large-scale blackouts due to long-term voltage instability has to be carefully assessed. To counteract critical network conditions, novel emergency control concepts acting timely and making use of available measures are essential for maintaining system stability in future power systems [1]. The major challenges for such systems are (i) a reliable reaction in due time, (ii) the adaptability in case of changing network conditions, (iii) the robustness to communication failures, and (iv) the coordinated activation of countermeasures.

As an alternative to centralized system integrity protection schemes requiring significant computational effort in case of largescale grids or the comprehensive installation of wide-area monitoring and control systems, as proposed in [2]. is proposed. Future substations will be connected through high speed communication links which allows for distributed controllers communicating between each other. In particular, a Multi-Agent System (MAS) is developed with intelligent agents situated at substations in the transmission and subtransmission network. Agents monitor the trend of local bus voltages, communicate with other agents and decide about corrective control actions autonomously. The developed approach allows for fast detection of an evolving voltage instability after the system is subjected to severe disturbances as well as the autonomous activation of countermeasures in order to restore a stable state of equilibrium with acceptable bus voltages.

The paper is structured as follows. First, in Section 2 the state of the art of voltage stability assessment and emergency control concepts against voltage collapse is presented. Section 3 gives an overview of the developed MAS including the algorithm for the assessment of voltages and the implemented emergency control scheme. Next, Section 4 outlines the scenario setup before in Section 5 the simulation results are presented. Finally, the paper closes with a conclusion and an outlook on future research in the field of (agent-based) emergency control systems for the prevention of voltage collapse (Section 6).

\section{Context and state of the art}

The industry approach for securing voltage stability in normal power system operation is often based on simple criteria: Voltage magnitudes are kept in pre-defined ranges and steady-state load flow based contingency analyses are performed with a manual selection and activation of countermeasures in case the $(\mathrm{N}-1)$ criterion is violated. Additionally, system protection schemes reacting to (major) disturbances are installed such as under-voltage load shedding relays.

In research, the assessment of long-term voltage stability, the computation of voltage stability margins and emergency control schemes have been intensively studied. Here, it is differentiated between distributed systems that solely rely on local measurements and centralized approaches based on comprehensive system information. One famous representative of centralized approaches is the continuation power flow, as first proposed in [3]. Another centralized control approach for the improvement of voltage stability has been proposed in [4] that is based on voltage-constrained optimal power flow. Yet, these approaches are designed for preventive control and are not applicable to corrective actions. In contrast to these, the authors in [5], [6] and [7] focus on corrective control of voltage instability that is performed centrally based on information obtained Phasor Measurement Units (PMUs) or based on model predictive control. General shortcomings of centralized approaches are their dependency on comprehensive and accurate system information and the thread of non-convergence in case of complex optimization problems. Some even require full observability of the network.

Distributed solutions for the local identification of voltage instability have been presented, e.g. by [8], [9] and [10]. Whereas the first are based on tracking the Thévenin equivalent as seen from a local bus, in [10] a control scheme has been developed that maintains power system voltages within pre-defined boundaries based on model predictive control and neighbour-to-neighbour communication between the controllers. Even if the controllers solve a local optimization based on limited system observability, the information that are exchanged serve as an input for the optimization problem. Further methods for voltage instability detection including both centralized and local approaches are summarized in [11] and [12]. 
This article has been accepted for publication in a future issue of this journal, but has not been fully edited.

Content may change prior to final publication in an issue of the journal. To cite the paper please use the doi provided on the Digital Library page.

Additionally, distributed agent-based solutions that solely rely on local measurements have been investigated. The general applicability of MAS for real-time control problems in electric power systems has been shown in [13]. A MAS-based solution that only relies on information measured locally or obtained from the neighbouring buses has been proposed by [14]. However, as the focus of the aforementioned optimal reactive power control approach is the minimization of real power losses and the improvement of voltage profiles, it is not suitable as an emergency control scheme. Another agent-based solution relying on distributed control and communication between neighbouring agents to counteract voltage instability is presented in [15]. However, agents need information about voltage angles that, under normal circumstances, cannot be provided without a comprehensive installation of PMUs. The authors in [16] propose an agent-based solution that performs calculation of the distance of the current operating point to the maximum loadability. Yet, the approach also requires information from PMUs and is based on steady-state load flow calculations. In [17], a MAS for emergency control against voltage collapse is proposed where agents coordinate different devices in post-fault situations. Yet, details on the algorithm for the identification of voltage instability are not presented. Another agent-based approach for power system restoration after a disturbance has been presented in [18]. In [19], an agent-based distributed reactive power management scheme has been presented whose purpose is to improve voltage stability of distribution systems based on inter-agent communication but is not applicable as an emergency control concept at transmission level. One main issue of distributed agent-based solution is the consensus problem which is defined as agents' need to agree on a common consent [20]. For agent-based control of emergency situations, it is therefore critical that the stabilization of the system is not prevented by the agents themselves. For this purpose, the MAS developed in this paper is not based on a negotiation between the agents which requires a positive outcome. Instead, agents follow their own goals and therewith enable reaching the main objective of stabilizing the power system.

In previous work the authors have developed an agent-based solution for the prevention of voltage collapse by a coordinated activation of available countermeasures [21]. In parallel, in [22] and [23] the Local Identification of Voltage Emergency Situations (LIVES) and extended-time LIVES (eLIVES) approaches have been presented for identifying voltage emergency situations solely based on local measurements gathered at Load Tap Changer (LTC) controlled buses. The main contribution of this paper is the extension of the aforementioned solutions by combining a robust algorithm for voltage stability assessment with a closed-loop logic for the execution of control actions in a MAS with a dedicated consideration of the inter-agent communication. As the approach solely relies on local measurements, it is real-time capable, robust with respect to communication failures and adaptive towards changing network conditions. Therefore, a fast and reliable identification of an impending longterm voltage instability is guaranteed by keeping the necessary data exchange between the agents to a minimum.

\section{Agent-based identification of voltage emergency situations}

The main objective of the developed MAS is the detection of voltage instability and the coordinated execution of emergency controls when the power system is close to voltage collapse. Here, intelligent agents are installed at each bus equipped with LTC in the transmission and/or subtransmission network, collect local measurements and communicate with surrounding agents.

As a main feature, the distributed agents do not need complete system information. Instead, they have a limited observability area that is characterized by its distance $d$. A distance $d=1$ indicates that the agent is able to communicate with its direct neighbours, whereas agents with a distance of $d=2$ are able to communicate with the neighbours of their neighbours. As the electric power system topology and the Information and Communication Technology (ICT) system are independent, the term distance only refers to the connection of the buses in the power system domain. For this,
Fig. 1 visualizes the topology of the MAS interacting with the power system and the ICT system. The MAS exchanges measurements and control signals with the power system. In the MAS domain agents send and receive messages. However, the physical agent communication is performed in the ICT domain where each agent is represented by one host. All hosts are connected via a router that routes the messages sent by the agents through the network according to existing standards. It is assumed that the outage of a single element in the power system domain does not interfere with the ICT system and vice versa.

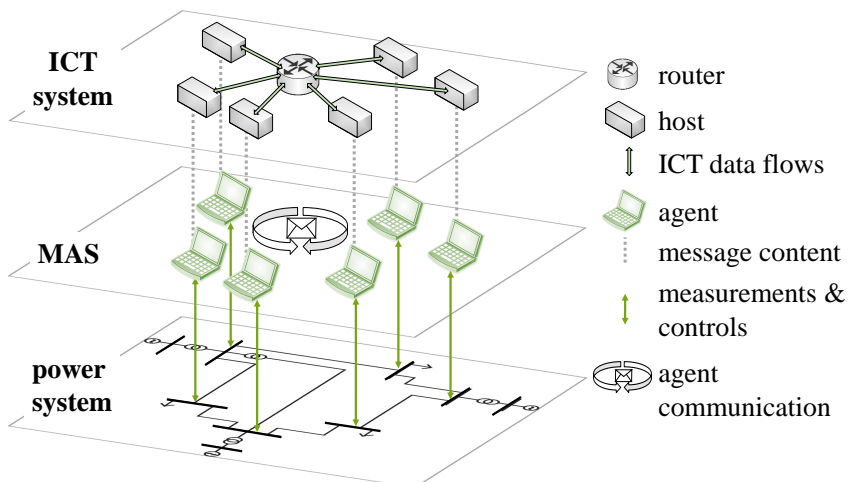

Fig. 1: Topology of MAS environment interacting with power and ICT system

\subsection{Local identification of voltage emergency condition}

The local identification of a voltage unstable situation is performed with a modified version of the eLIVES algorithm presented in [23]. The monitoring of the system response is activated in case of LTC tap adjustment. In order to detect an evolving voltage instability, eLIVES observes the voltage evolution during the restoration and analyses the overall trend of the voltage. The algorithm uses only locally available measurements and information, namely the distribution voltage at the Medium Voltage (MV) side of the transformer and the tap ratio. The proposed algorithm accommodates variable tap delays and is robust to measurement noise on the input signal, as demonstrated in [23].

3.1.1 Recursive least square fitting of voltage measurements: The local voltage assessment is executed when the distribution voltage $v_{\mathrm{mv}}$ leaves the deadband and the LTC is still active, i.e. it has not yet reached its tap limits. After the first tap change has been detected at time $t_{\mathrm{rec}}$, the method starts recording voltage measurements. These measurements are utilized to identify the trend of the voltage evolution $v_{\text {fit }}$ using a linear regression model:

$$
v_{\mathrm{fit}}(t)=m_{t} \cdot t+b_{t}
$$

In each time step $t$, the estimator determines the parameters $m_{t}$ and $b_{t}$. Since the method assesses long-term voltage stability, which may require several minutes of monitoring after a disturbance, a recursive least squares fitting is used [24].

3.1.2 Rules for raising a local warning: When a sufficient number of samples is collected, the slope $m_{t}$ is evaluated. A negative slope indicates that the voltage magnitude is declining and i.e. load voltage (and hence power) cannot be restored to the LTC setpoint while a positive slope suggests an overall recovery of the LTC controlled voltage. In case the current slope $m_{t}$ is negative, it is compared to the negative slope $m_{t-1}$, which was computed at the previous discrete time step. A warning is issued, if $m_{t}$ is more negative than $m_{t-1}$. For improving the performance of the method, the first evaluation of the slope is delayed by $t_{\text {wait }}$. The delay was chosen to give the LTC time to restore the voltage and to allow revelation of the overall voltage trend. Further, it makes the algorithm 
This article has been accepted for publication in a future issue of this journal, but has not been fully edited.

Content may change prior to final publication in an issue of the journal. To cite the paper please use the doi provided on the Digital Library page.

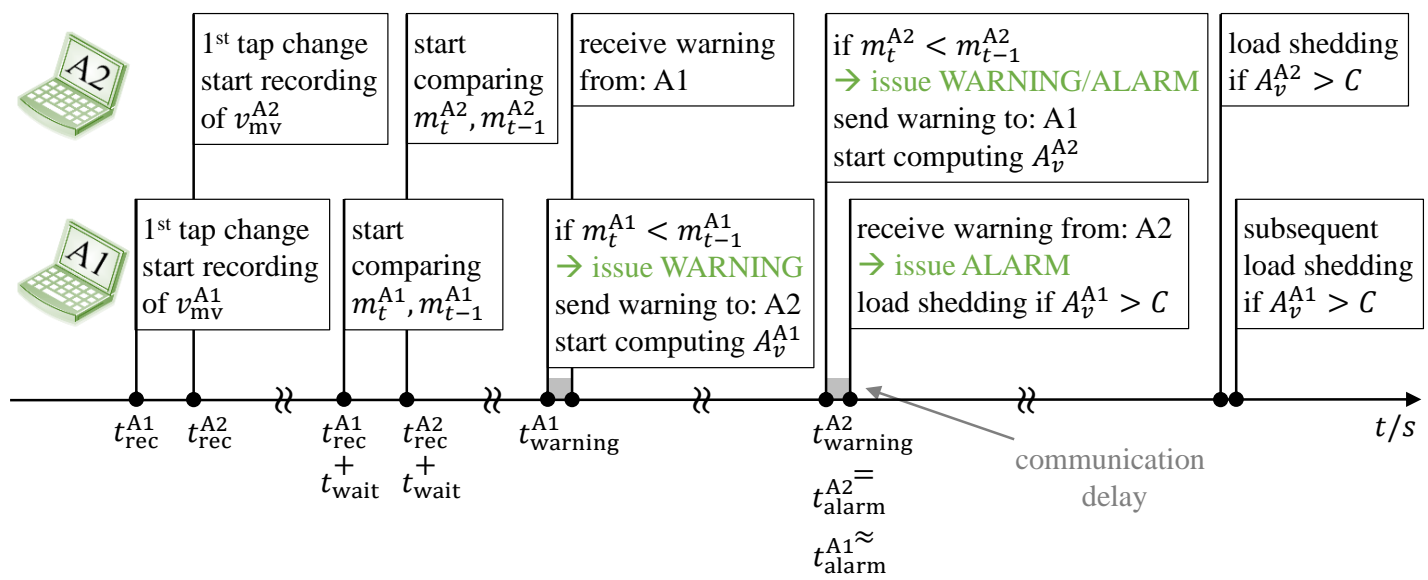

Fig. 2: Sequence of events in the agent-based identification and control of voltage emergency situations (example with two agents)

more robust to data uncertainty. The monitoring and assessment of voltages is stopped in two conditions:

1. $v_{\mathrm{mv}}$ was restored by the respective LTC, re-entered the deadband and remained inside longer than a pre-set time period, or

2. the LTC reaches its tap limit.

The first indicates a recovery of the distribution voltage. As voltage stability is driven by the effect of the local LTC in combination with all voltage controllers in the system, voltage control is lost at the particular bus in case the LTC exhausts its tap range which should not be interpreted as instability.

\subsection{Rules for raising an alarm}

Taking advantage of the communication between the distributed agents, a situation is assumed to be critical if two or more neighbouring agents raise a warning. This means that agents issuing a warning need a confirmation from at least one of the surrounding agents until they raise an alarm and are able to perform control actions. Thus, an (incorrect) detection of a single agent does not result in the activation of countermeasures. This principle is visualized in Fig. 2.

The process starts with the first tap change at $t_{\text {rec }}^{\mathrm{A} 1}$ for the first, respectively $t_{\mathrm{rec}}^{\mathrm{A} 2}$ for the second agent in this example. After an additional waiting period $t_{\text {wait }}$, the agents compare their respective voltage slopes $m_{t}$ and $m_{t-1}$. Assuming agent A1 detects an increasingly negative slope at $t_{\mathrm{w} 1}^{\mathrm{A} 1} \mathrm{Arning}$, it sends a warning message to all agents within its observability area. Thus, agent A1 waits for a confirmation from surrounding agents and starts preparing for executing control actions by computing the voltage deviation integral $A_{v, t}$ (see Section 3.3). Hence, the preparation starts before the agent receives a confirmation, still the execution is delayed until the reception. As soon as another agent, e.g. A2, identifies an increased negative slope at $t_{\mathrm{warning}}^{\mathrm{A} 2}$, it also raises a warning and sends a message to all of its surrounding agents. Thus, both agents issue an alarm at $t_{\text {alarm }}^{\mathrm{A} 2}=t_{\text {warning }}^{\mathrm{A} 2} \approx t_{\text {alarm }}^{\mathrm{A} 1}$. In this process, the order of sending and receiving the warning message is irrelevant. However, agents that did not raise a warning themselves do not participate in the execution of control actions. Even if this process causes a delay on the first agent detecting a voltage critical situation as it is slowed down until it receives a confirmation, the confirmation process decreases the risk of false alarms. Moreover, the implemented load shedding strategy detailed in Section 3.3 makes critical agents act first.

One main feature of the MAS is its robustness towards single or even multiple communication failures. As voltage stability affects several nodes in a region, it is assumed that another agent can distribute the required confirmation message in case a single communication path between two agents is disturbed and the respective warning message is not received. Furthermore, neighbouring agents can also take over in performing the required control actions in order to keep the operability of the whole system. However, in order to make the MAS robust towards severe communication failures, e.g., an outage of the complete ICT infrastructure, a local backup solution is implemented. For this, the agent monitors the transmission voltage at the High Voltage (HV) side of the transformer $v_{\mathrm{hv}}$.

$$
\text { If } v_{\mathrm{hv}}<v_{\mathrm{hv}, \text { alarm }} \text { for } \Delta t_{\mathrm{hv}, \text { alarm }}
$$

the agent is allowed to perform emergency control actions immediately without the confirmation from one of the surrounding agents. $v_{\text {hv,alarm }}$ is the corresponding voltage threshold whereas $\Delta t_{\mathrm{hv}, \text { alarm }}$ is a pre-set time interval in which the voltage needs to stay below the threshold. In order to prevent the backup system from premature actions, $v_{\mathrm{hv} \text {,alarm }}$ needs to be set conservatively. Yet, it cannot be too low in order to allow execution of control actions in due time and thus avoid occurrence of unacceptably low voltages, which may trigger (under-voltage) protection equipment. Commonly, $v_{\mathrm{hv} \text {,alarm }}$ is in a range of 0.85 to 0.9 p.u.. Once an $\mathrm{HV}$ alarm is issued, the value of $v_{\mathrm{hv}}$,alarm is stored for subsequent control actions.

\subsection{Emergency controls to stabilize the system}

If an agent raises an alarm, after both the local identification of an increasingly negative slope and the reception of a confirmation message from one of the surrounding agents, the agent activates the emergency control scheme. As the MAS serves as an emergency control system against voltage collapse, the agents perform load curtailment as this is a reliable and suitable countermeasure to counteract voltage instability and regain a steady-state of equilibrium [25]. Here, blocks of load are shed in several steps until stability is restored. The load shedding strategy is designed to first shed load at the most critical bus. For this purpose, the concept of [26] was applied, where the time delay and the amount of load shedding were adjusted according to the disturbance severity. In contrast to this reference, the MAS only implements the time delay whereas the amount of load shedding is fixed. Without the implementation of a delay, multiple agents would react instantaneously after the reception of a warning from the surrounding agents (provided they have already detected a critical situation and have not yet received a confirmation).

Thus, the process for the load shedding is as follows: First, an agent stores its transmission voltage $v_{\mathrm{hv} \text {, warning }}=v_{\mathrm{hv}}\left(t_{\mathrm{warning}}\right)$ at the moment it raises the warning $t_{\text {warning. This value serves as a }}$ threshold for successive load curtailments. Notably, the preparation for the load shedding starts before the reception of a confirmation in order to enable that the most critical agents act first (see. Section 3.2 and Fig. 2). Next, at time $t_{*}=t_{\text {warning }}$ the agent starts computing the integral of the voltage deviation $A_{v, t}$ at its bus over the time interval $\tau$ : 
This article has been accepted for publication in a future issue of this journal, but has not been fully edited.

Content may change prior to final publication in an issue of the journal. To cite the paper please use the doi provided on the Digital Library page.

$$
A_{v, t}=\int_{t_{*}}^{t_{*}+\tau}\left(v_{\mathrm{hv}, \text { warning }}-v_{\mathrm{hv}}(t)\right) \mathrm{d} t .
$$

As the MAS acts in discrete time steps, Eq. (3) is modified in order to fit for a discrete control system. Here, the voltage time deviation $A_{v, t}$ is updated in every time step $t$ starting at time $t_{*}=t_{\text {warning: }}$ :

$$
A_{v, t}=A_{v, t-1}+\left(t-t_{t-1}\right) \cdot\left(v_{\mathrm{hv}, \text { warning }}-v_{\mathrm{hv}}(t)\right) .
$$

As soon as $A_{v, t}$ reaches the pre-set value $C$ (and additionally the agent has received a warning message from one of the neighbouring agents), it sheds one block of load and $A_{v, t}$ is reset to zero. As long as $v_{\mathrm{hv}}$ stays below $v_{\mathrm{hv}}$,warning, the process is repeated, i.e. the agent can subsequently shed further blocks of load. Thus, the control operates in closed-loop, which further contributes to the overall robustness of the scheme [26]. This algorithm also exhibits an inverse time characteristic. Namely, the deeper and faster the voltage $v_{\text {hv }}$ declines, the less time it takes for the integral to reach value $C$. Note finally that, if the agent has not received a confirmation from surrounding agents by the moment the integral reaches the value $C$, it will wait until it either receives a confirmation or the local backup alarm is raised.

\section{Scenario setup}

The agent-based identification and control of voltage emergency situations has been implemented in the Java Agent DEvelopment (JADE)-framework which provides an environment for the modelling and simulation of agents including their administration and a messaging service. In order to demonstrate the performance of the MAS, a co-simulation environment has been set up interfacing JADE with DIgSILENT PowerFactory, which runs time-domain simulations of the power system. For the agent communication the built-in

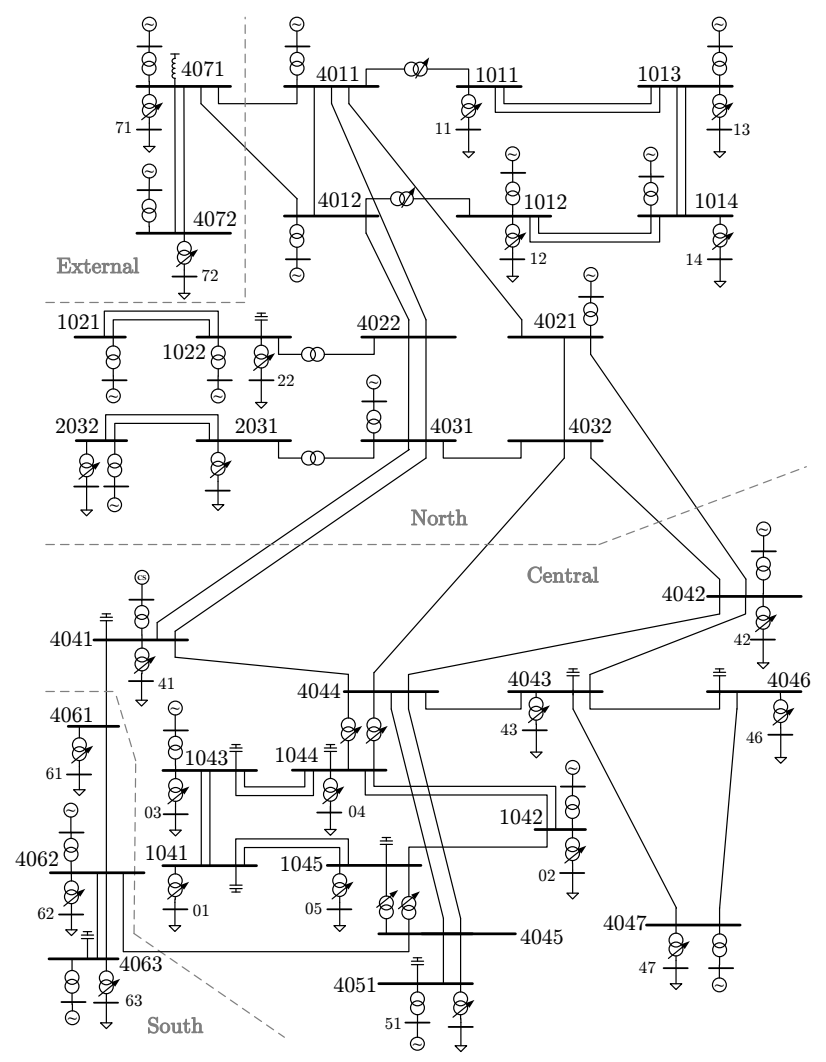

Fig. 3: Topology of the IEEE Nordic Test System (based on [25])
JADE-communication has been used. The power system is simulated with a time step size of $10 \mathrm{~ms}$. Yet, the agents do not act in every time step but in discrete steps of $500 \mathrm{~ms}$ which is sufficient for long-term voltage stability.

In order to demonstrate the applicability of this approach, simulations were carried out on the Nordic Test System detailed in [25], whose network topology is shown in Fig. 3. Here, the behaviour of the distribution network is represented by a voltage sensitive load and a distributed generating unit in parallel. Whereas loads are modelled using the well-known exponential load model and parametrized according to [25], distributed generators can be either operated with constant power factor or a $Q(V)$-characteristic, as e.g. presented in [27]. Between $v=0.96$ and 1.04 p.u. the reactive power output of the generating unit is adjusted proportionally to the voltage deviation with a deadband between $v=0.995$ and 1.005 p.u.. Notably, distributed generators are only located in the Central area. For the load shedding a fixed block size of $\Delta P_{\mathrm{LS}}=25 \mathrm{MW}$ was chosen. $t_{\mathrm{wait}}$ is $20 \mathrm{~s}$, whereas $v_{\mathrm{hv} \text {,alarm }}$ and $t_{\mathrm{hv} \text {,alarm }}$ were set to $0.9 \mathrm{p} . \mathrm{u}$. and $3 \mathrm{~s}$, respectively. $C=3 \mathrm{~s} \cdot 0.02 \mathrm{p} . \mathrm{u} .=0.06 \mathrm{p} . \mathrm{u} . \cdot \mathrm{s}$.

For analysing the performance of the MAS in stressed conditions, the initial situation is highly loaded and not in an (N-1)-secure state [25]. Then, a short circuit occurs on transmission line 4032-4044 at $t_{\text {fault }}=1 \mathrm{~s}$. The fault is cleared through tripping of the respective transmission line $\left(t_{\text {out }}=1.1 \mathrm{~s}\right)$. Long-term voltage instability is driven by LTCs trying to bring the distribution voltages back in their deadbands $[0.99,1.01]$ p.u. and over excitation limiters controlling the field current of generators. The system experiences voltage collapse in the Central region after around $180 \mathrm{~s}$. Transmission voltages in the Northern and Southern region stay almost unaffected.

\section{$5 \quad$ Simulation results}

Table 1 summarizes the simulations reported in this section. First, solely the assessment of voltages is performed and no emergency control is applied, in order to investigate the impact of the size of the observed area $d$ on the performance of the algorithm. Next, emergency controls are executed by the distributed agents with a varying available flexibility defined by the minimum load $P_{\mathrm{L}, \min }$ that needs to stay connected to an agent's node with $P_{0}$ being the initial load consumption. Further, the performance of the algorithm is investigated in case distributed generating units in the underlying network support distribution voltages by feeding-in reactive power. Finally, the robustness with respect to communication failures is simulated.

\subsection{Assessment of voltages with variation of the size of the observability area}

Figure 4 shows the evolution of LTC tap ratios as well as transmission and distribution voltages of selected transformers in the

\begin{tabular}{|c|c|c|c|c|c|}
\hline scenario & $\begin{array}{c}\text { load } \\
\text { shedding }\end{array}$ & $d$ & $P_{\mathrm{L}, \min }$ & $\begin{array}{c}\text { ICT } \\
\text { failure }\end{array}$ & $\begin{array}{l}\text { reactive power } \\
\text { strategy of DGs }\end{array}$ \\
\hline A. 1 & 0 & 1 & - & - & $\cos \varphi=1$ \\
\hline A. 2 & 0 & 2 & - & - & $\cos \varphi=1$ \\
\hline A. 3 & 0 & 3 & - & - & $\cos \varphi=1$ \\
\hline B. 1 & 1 & 1 & 0 & - & $\cos \varphi=1$ \\
\hline B. 2 & 1 & 1 & $0.85 P_{0}$ & - & $\cos \varphi=1$ \\
\hline B.3 & 1 & 1 & $0.90 P_{0}$ & - & $\cos \varphi=1$ \\
\hline C.1 & 0 & 1 & - & - & \multirow{4}{*}{$\begin{array}{l}Q(V) \text { with } \\
Q_{\text {flex }}=100 \text { Mvar } \\
Q(V) \text { with } \\
Q_{\text {flex }}=100 \text { Mvar } \\
Q(V) \text { with } \\
Q_{\text {flex }}=50 \text { Mvar } \\
Q(V) \text { with } \\
Q_{\text {flex }}=50 \text { Mvar }\end{array}$} \\
\hline C.2 & 1 & 1 & 0 & - & \\
\hline C.3 & 0 & 1 & - & - & \\
\hline C.4 & 1 & 1 & 0 & - & \\
\hline D. 1 & 1 & 1 & 0 & $1045-1041$ & $\cos \varphi=1$ \\
\hline D.2 & 1 & 1 & 0 & $4043-4042$ & $\cos \varphi=1$ \\
\hline D.3 & 1 & 1 & 0 & complete & $\cos \varphi=1$ \\
\hline
\end{tabular}

Table 1 Overview of simulations 
This article has been accepted for publication in a future issue of this journal, but has not been fully edited.

Content may change prior to final publication in an issue of the journal. To cite the paper please use the doi provided on the Digital Library page.

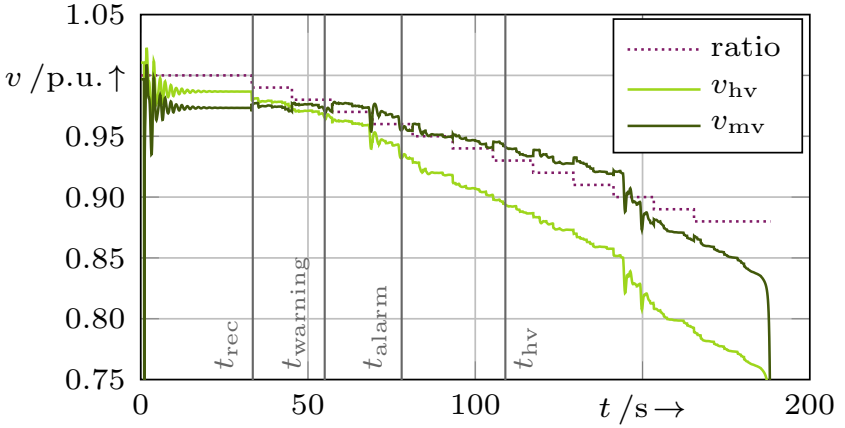

$a$

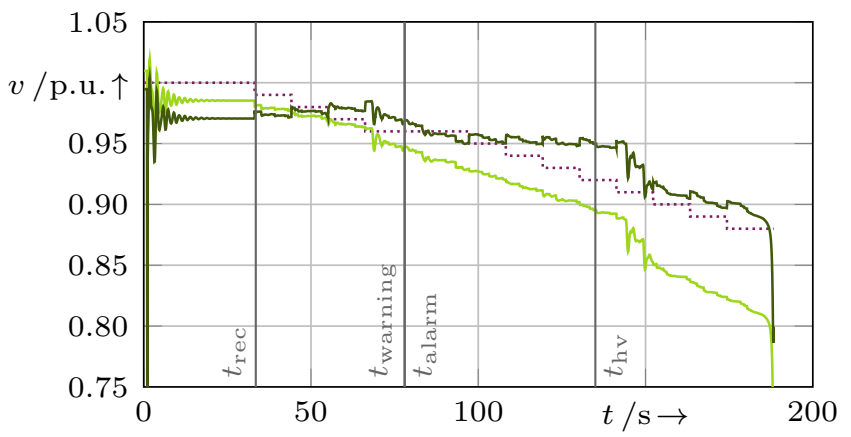

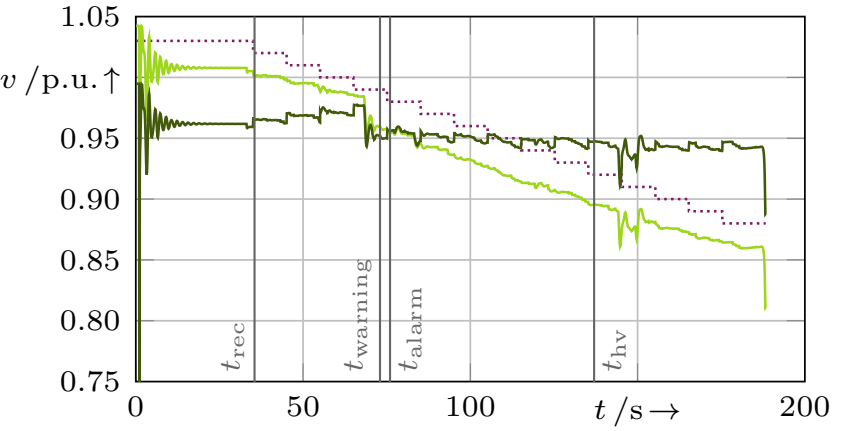

$b$

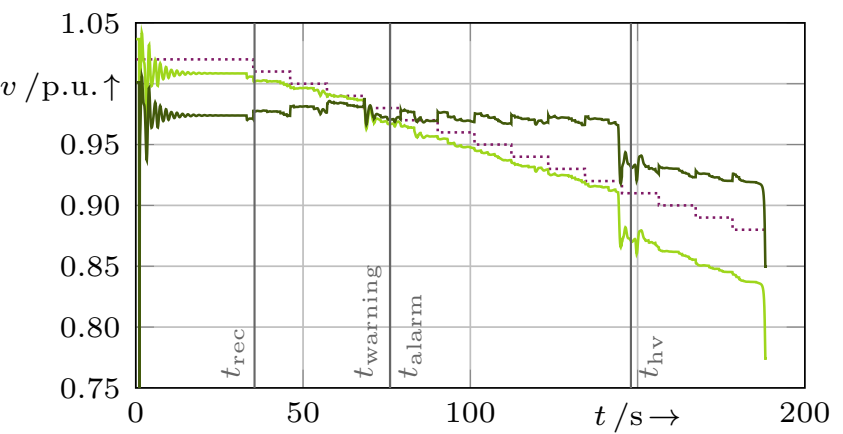

$d$

Fig. 4: Evolution of transmission ( $\left.v_{\mathrm{hv}}\right)$, distribution voltages $\left(v_{\mathrm{mv}}\right)$ and ratios of selected transformers in Scenario A.1

$a$ LTC-1041-01

$b$ LTC-4042-42

$c$ LTC-1045-05

$d$ LTC-4043-43

Central region in Scenario A.1. Agents start recording voltages at the time of their first tap change at $t_{\mathrm{rec}}^{1041}=t_{\mathrm{rec}}^{1045}=33.0 \mathrm{~s}$ and $t_{\mathrm{rec}}^{4042}=t_{\mathrm{rec}}^{4043}=35.5 \mathrm{~s}$. At $t=t_{\mathrm{rec}}+t_{\mathrm{wait}}$ they start the assessment of the voltage slope $m_{t}$. Agent 1041 is the first issuing a warning at $t_{\text {warning }}^{1041}=55.5 \mathrm{~s}$ after identifying the negative trend of the voltage evolution (see Fig. 4a) whereas agent 1045 raises its warning at $t_{\text {warning }}^{1045}=77.9 \mathrm{~s}$ (see Fig. 4c). As agent 1041 has already sent a warning message to agent 1045 , both agents issue an alarm at

$$
t_{\mathrm{alarm}}^{1041}=t_{\mathrm{alarm}}^{1045}=t_{\mathrm{warning}}^{1045}=77.9 \mathrm{~s} .
$$

The agents 4042 and 4043 do not interact with the agents 1041 and 1045 due to the distance of $d=1$. Agent 4042 is the first of the two raising a warning at $t_{\text {warning }}^{4042}=72.9 \mathrm{~s}$. Once agent 4043 issues a warning at

$$
t_{\mathrm{alarm}}^{4042}=t_{\mathrm{alarm}}^{4043}=t_{\mathrm{warning}}^{4043}=76.4 \mathrm{~s}
$$

both agents raise the alarm (see Fig. $4 b, d$ ). Furthermore, the time of

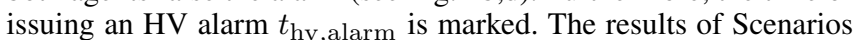
A. 1 to A. 3 are summarized in Table 2 .

In all three scenarios, warnings were raised at buses that are mainly located in the Central region. As the raise of a warning is solely based on the assessment of local voltage measurements, the time of issuing the warning is independent from the size of the observability area. The same applies for the HV alarms that have been exclusively issued in the Central region where the most severe voltage drop has been experienced. Yet, the size of the observability area determines the number of agents an agent is able to communicate with, and thus influences the time of receiving a confirmation message and therewith the time of raising an alarm. However, it can be seen that increasing distance $d$ has low impact on $t_{\text {alarm }}$. There is no difference between $d=2$ and $d=3$. A difference can be observed for agent 1044 that receives its confirmation message earlier in case of $d>1$ as well as the agents at nodes 4051 and 4062 that only raise an alarm in case of $d>1$. Remote agents issuing a warning, e.g., agent 1022, do not get a confirmation in any scenario. This is desirable since the Central region is known to be more affected to instability and to be the place where load shedding is the most effective [25].

\subsection{Applying emergency controls with varying flexibility}

Since an increase of the observed area does not impact the instability detection time at the most critical buses (e.g. 1041, 1045, 4042 and 4043), only a distance $d=1$ is simulated in the following. Figure 5 shows the evolution of transmission and distribution voltages of the transformer connected to bus 1041 and feeding the MV bus 01 as well as its ratio in Scenarios B.1 and B.3 . Further, the power consumption of relevant loads in the Central region is visualized. Here, the active power is normalized $\left(p=P(t) / P_{0}\right)$. Steps of load shedding are marked with grey arrows.

It can be seen that voltages recover after the load curtailment in an acceptable range in both scenarios (see Fig. 5a,b). For Scenario

Table 2 Results of assessment of voltage measurements in Scenarios A.1 to A.3

\begin{tabular}{lrrrrrc}
\hline region & \multirow{2}{*}{ bus } & $\begin{array}{c}\text { time of } \\
\text { warning }\end{array}$ & \multicolumn{1}{c}{ A.1 } & \multicolumn{1}{c}{ time of alarm } & \multicolumn{1}{c}{ A.3 } & time of \\
& & HV alarm & (77.9 & 77.9 & 77.9 & 109.3 \\
\hline Central & 1041 & 55.5 & 769.1 & 169.1 & 169.1 & 152.2 \\
Central & 1042 & 169.1 & 169.1 & 105.8 & 105.8 & 125.2 \\
Central & 1043 & 105.8 & 105.8 & 105.9 & 113.3 \\
Central & 1044 & 77.9 & 105.8 & 77.9 & 77.9 & 134.2 \\
Central & 1045 & 77.9 & 77.9 & 77.9 & 77.9 & - \\
North & 1022 & 94.3 & - & - & - & 137.2 \\
Central & 4042 & 72.9 & 76.4 & 76.4 & 76.4 & 136.2 \\
Central & 4043 & 76.4 & 76.4 & 76.4 & 76.4 & 148.2 \\
Central & 4046 & 80.3 & 80.3 & 80.3 & 80.3 & 148.2 \\
Central & 4047 & 186.0 & 186.0 & 186.0 & 186.0 & 168.6 \\
Central & 4051 & 156.1 & - & 156.1 & 156.1 & 175.1 \\
South & 4062 & 129.2 & - & 129.2 & 129.2 & - \\
\hline
\end{tabular}


This article has been accepted for publication in a future issue of this journal, but has not been fully edited.

Content may change prior to final publication in an issue of the journal. To cite the paper please use the doi provided on the Digital Library page.

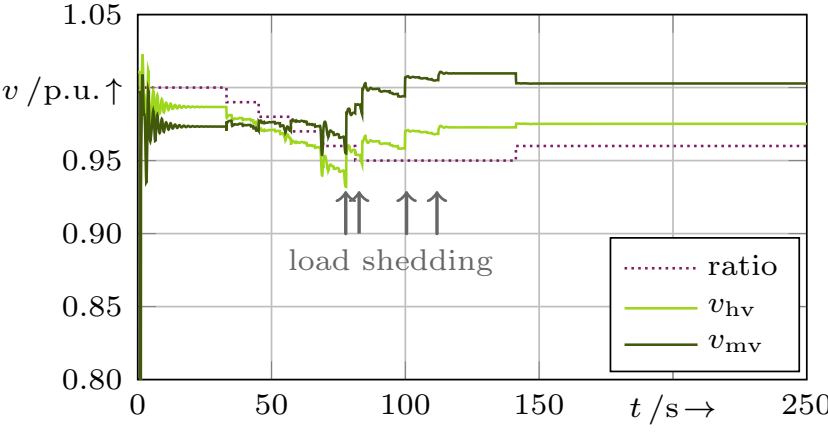

$a$

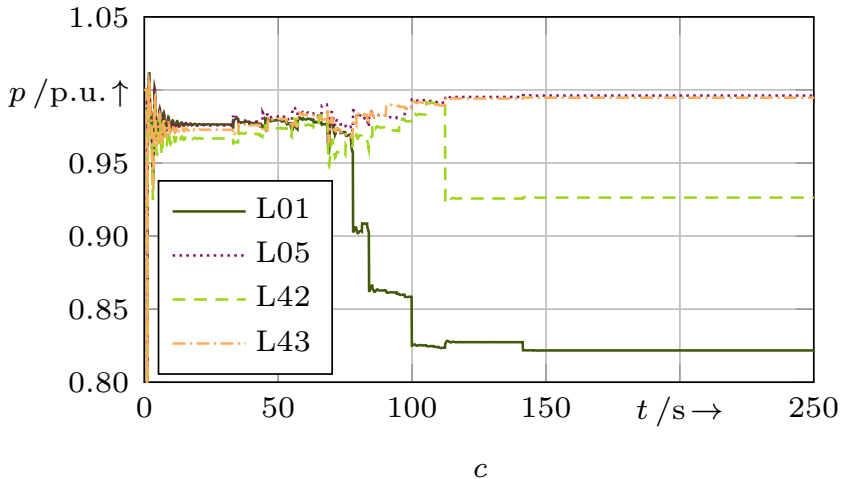

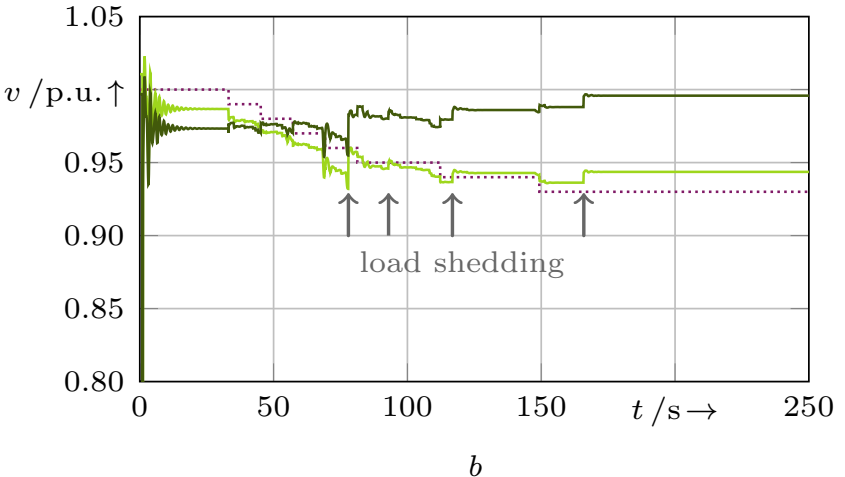

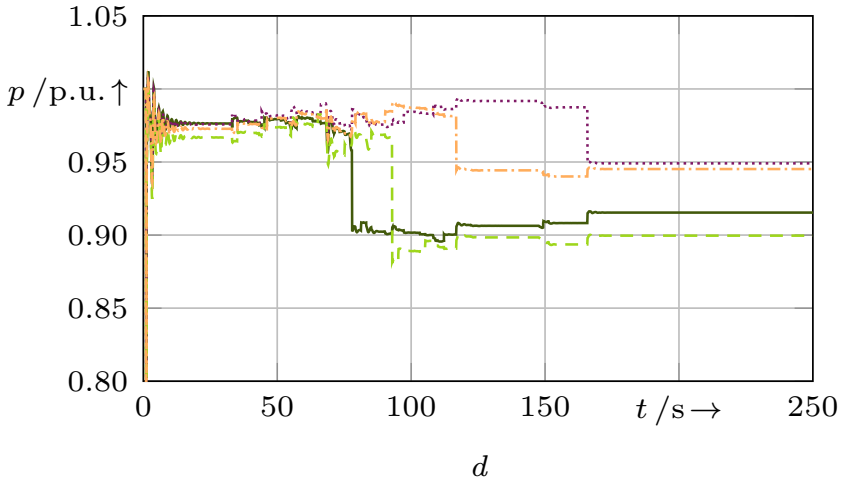

Fig. 5: Evolution of transmission ( $\left.v_{\mathrm{hv}}\right)$, distribution voltages ( $\left.v_{\mathrm{mv}}\right)$ and ratio of transformer 1041-01 as well as active power consumption of relevant loads in Scenario B.1 and B.3

a Scenario B.1 - Voltages and tap ratio

$b$ Scenario B.3 - Voltages and tap ratio

$c$ Scenario B.1 - Power consumption

$d$ Scenario B.3 - Power consumption

B.1, solely agents 1041 and 4042 participate in the load curtailment (see Fig. 5c). As 1041 is the most critical bus raising the warning first and having the most severe voltage fall, $75 \mathrm{MW}$ of load are shed at this bus, whereas agent 4042 sheds only one block of $25 \mathrm{MW}$. In Scenario B.3, as can be seen in Fig. 5d, the amount of load shedding is equally spread over the agents 1041, 1045, 4042 and 4043 each curtailing $25 \mathrm{MW}$. Indeed, due to $P_{\mathrm{L}, \min }$ set to $90 \%$ of the initial load consumption $P_{0}$, each agent is only allowed to shed one block of load.

Nevertheless, this demonstrates that even in case of agents having low available flexibility, surrounding agents take over and perform the required load curtailment. However, the stabilized transmission voltages settle at lower values in Scenario B.3 (see Fig. 5b) compared to Scenario B.1 (see Fig. 5a). Table 3 summarizes the results of the Scenarios B.1, B.2 and B.3 including the time of raising a warning or an alarm, the stabilized transmission voltage $v_{\mathrm{hv}, \mathrm{s}}$ as well as the total amount of load shedding $\Delta P_{\mathrm{LS}}$.

Table 3 Results of assessment of voltage measurements and amount of load shedding in Scenarios B. 1 to B.3 (time values marked with * indicate identification solely in scenario B.3)

\begin{tabular}{ccccccccc}
\hline \multirow{2}{*}{ bus } & time of & time of & \multicolumn{2}{c}{ B.1 } & \multicolumn{2}{c}{ B.2 } & \multicolumn{2}{c}{ B.3 } \\
& warning & alarm & $\Delta P_{\mathrm{LS}}$ & $v_{\mathrm{hv}, \mathrm{s}}$ & $\Delta P_{\mathrm{LS}}$ & $v_{\mathrm{hv}, \mathrm{s}}$ & $\Delta P_{\mathrm{LS}}$ & $v_{\mathrm{hv}, \mathrm{s}}$ \\
\hline 1041 & 55.5 & 77.9 & 75 & 0.975 & 50 & 0.956 & 25 & 0.944 \\
1044 & 77.9 & - & - & 0.944 & - & 0.935 & - & 0.934 \\
1045 & 77.9 & 77.9 & - & 0.960 & - & 0.950 & 25 & 0.950 \\
1022 & $113.8^{*}$ & - & - & 1.016 & - & 1.013 & - & 1.015 \\
4042 & 72.9 & 76.4 & 25 & 0.963 & 25 & 0.956 & 25 & 0.958 \\
4043 & 76.4 & 76.4 & - & 0.974 & 25 & 0.967 & 25 & 0.969 \\
4051 & $104.8^{*}$ & - & - & 1.035 & - & 1.030 & - & 1.030 \\
\hline
\end{tabular}

\subsection{Assessment of emergency control in case of local voltage support at distribution level}

In scenarios C. 1 to C.4, equivalent generators representing distributed generators located in the distribution network are operated with a $Q(V)$-characteristic, therewith increasing the reactive power output with decreasing distribution voltages. The generators are equivalents replacing a large number of small units. Two different flexibility scenarios have been simulated. For the first, the equivalent generating units at each bus have a flexibility of $Q_{\text {flex }}=100$ Mvar whereas for the second case it is reduced to $Q_{\text {flex }}=50$ Mvar. In Scenarios C. 1 and C.3 no emergency control is applied, while in Scenarios C. 2 and C. 4 the agents are able to perform load shedding. Figure 6 shows transmission and distribution voltages of the transformers connected to bus 1041 and 4042, their tap ratios and

Table 4 Results of assessment of voltage measurements and amount of load shedding in Scenarios C. 1 to C.4 (time values marked with $*$ indicate identification in Scenario C.2 and C.4 respectively)

\begin{tabular}{|c|c|c|c|c|c|c|c|c|}
\hline \multirow[b]{2}{*}{ bus } & \multicolumn{2}{|c|}{ C.1/C.2 } & \multirow{2}{*}{$\begin{array}{c}\text { C.1 } \\
\text { time of } \\
\text { hv alarm }\end{array}$} & \multirow{2}{*}{$\begin{array}{c}\text { C.2 } \\
\Delta \\
P_{\mathrm{LS}}\end{array}$} & \multicolumn{2}{|c|}{ C.3/C.4 } & \multirow{2}{*}{$\begin{array}{c}\text { C.3 } \\
\text { time of } \\
\text { hv alarm }\end{array}$} & \multirow{2}{*}{$\begin{array}{c}\text { C.4 } \\
\Delta \\
P_{\mathrm{LS}}\end{array}$} \\
\hline & $\begin{array}{c}\text { time of } \\
\text { warning }\end{array}$ & $\begin{array}{l}\text { time of } \\
\text { alarm }\end{array}$ & & & $\begin{array}{c}\text { time of } \\
\text { warning }\end{array}$ & $\begin{array}{l}\text { time of } \\
\text { alarm }\end{array}$ & & \\
\hline 1041 & $115.9^{*}$ & $122.4^{*}$ & 206.6 & 50 & $89.4^{*}$ & $89.4^{*}$ & 161.7 & 25 \\
\hline 1042 & 215.6 & 215.6 & 225.1 & - & 182.1 & 182.1 & 185.6 & - \\
\hline 1043 & 170.7 & 170.7 & 215.1 & - & 122.3 & 122.3 & 173.6 & - \\
\hline 1044 & 139.8 & 170.7 & 181.2 & - & $76.4^{*}$ & 122.3 & 147.7 & - \\
\hline 1045 & $122.4^{*}$ & $122.4^{*}$ & 204.6 & 50 & $88.4^{*}$ & $89.4^{*}$ & 168.7 & 25 \\
\hline 2031 & 163.7 & - & - & - & 101.9 & - & 277.8 & - \\
\hline 4041 & 194.2 & 194.2 & - & - & - & - & 293.7 & - \\
\hline 4042 & 167.8 & 167.8 & 179.7 & - & $83.9^{*}$ & $83.9^{*}$ & 157.2 & 25 \\
\hline 4043 & $75.9^{*}$ & 167.3 & 202.6 & - & $80.9^{*}$ & 83.9* & 175.1 & 25 \\
\hline 4046 & 167.3 & 167.3 & 202.6 & - & 126.3 & 126.3 & 175.1 & - \\
\hline 4047 & 205.1 & 205.1 & 234.5 & - & 255.4 & 255.4 & 195.6 & - \\
\hline 4051 & $140.8^{*}$ & - & 288.9 & - & 252.4 & - & 232.9 & - \\
\hline 4061 & 184.7 & 184.7 & - & - & - & - & - & - \\
\hline
\end{tabular}


This article has been accepted for publication in a future issue of this journal, but has not been fully edited.

Content may change prior to final publication in an issue of the journal. To cite the paper please use the doi provided on the Digital Library page.

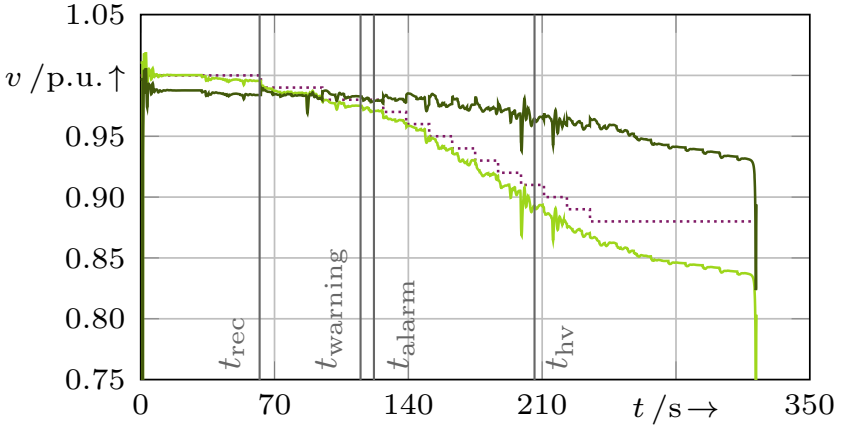

$a$

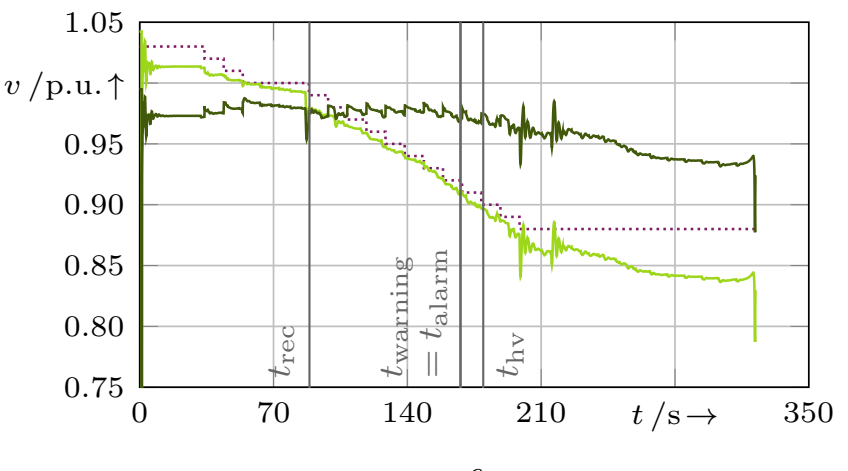

$c$
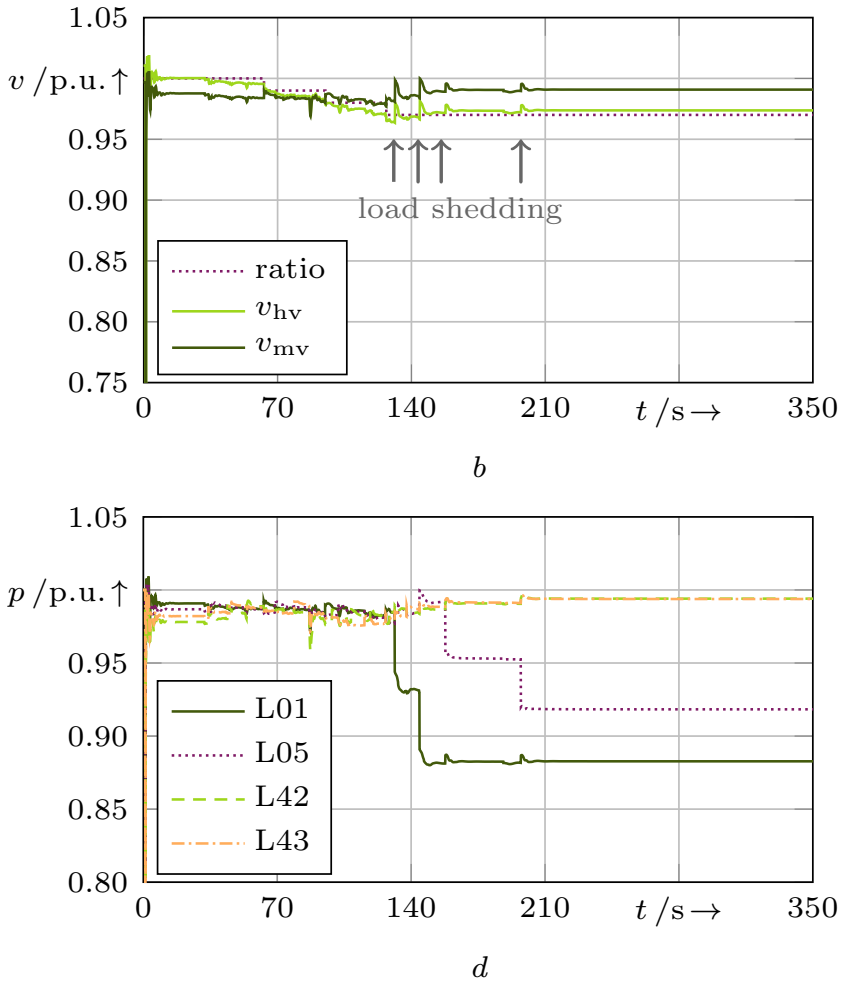

Fig. 6: Evolution of transmission $\left(v_{\mathrm{hv}}\right)$, distribution voltages $\left(v_{\mathrm{mv}}\right)$ and ratio of selected transformers as well as active power consumption of relevant loads in Scenario C.1 and C.2

a Scenario C.1 - Voltages and tap ratio of LTC-1041-01

$b$ Scenario C. 2 - Voltages and tap ratio of LTC-1041-01

$c$ Scenario C.1 - Voltages and tap ratio of LTC-4042-42

$d$ Scenario C.2 - Power consumption

the normalized active power consumption of relevant loads in the Central region in Scenarios C.1 and C.2. Due to the reactive power support of distributed generators, distribution voltages can be kept in an acceptable range for a longer time until voltage collapse occurs (see Figs. 6a and c). Therewith, the time for raising a warning or an alarm is delayed. As can be seen when comparing Fig. 6a to Fig. 4a, the tap adjustment of LTC-1041-01 is also delayed, yet not avoided. Notably, even if the first tap change of transformer 4042-42 takes place at $33.8 \mathrm{~s}$, the start of the recording of the voltage slope is delayed as the distribution voltage re-enters the deadband $[0.99,1.01]$ p.u. at around $56 \mathrm{~s}$. Nevertheless, the implemented algorithm is able to identify the evolving voltage instability and apply emergency controls in due time (see Fig. 6d) with an equal amount of shed load compared to Scenario B.1. The results for the Scenarios C. 1 to C. 4 are summarized in Table 4 .

\subsection{Robustness to communication failures}

Figure 7 shows the transmission and distribution voltages of the transformer connected to bus 1041 feeding MV bus 01, its tap ratio and the normalized active power consumption of relevant loads in the Central region in Scenarios D.1 and D.3. Further, Table 5 summarizes the results for all three scenarios including the assessment of voltages, the amount of load shedding and the stabilized transmission voltages. In Scenario D. 1 it is assumed that the confirmation message from agent 1045 to agent 1041 is not delivered, whereas in Scenario D.2 agent 4042 does not receive the message from agent 4043. Hence, in both cases, the respective agent does not participate in emergency control actions but the surrounding agents take over. However, as the load curtailment is delayed, voltages continue to decline and more agents detect critical voltage deviations $\left(A_{V}>C\right)$ at their respective nodes. Therefore, more load is shed compared to the simulations in Section 5.2. Nevertheless, in both scenarios the local backup criterion is not violated. This indicates, that the proper operation of the proposed scheme is guaranteed in case of single communication failures. However, a complete outage of the ICT system as simulated in Scenario D. 3 disables the whole interagent communication. As a result, the agents' local backup criterion is activated when voltages continue to decline, and load shedding is performed later (see Fig. 7b,d). Thus, voltages recover at lower values (see Table 5) as the voltage threshold for load shedding is now $v_{\text {hv,alarm }}=0.9$ p.u. $<v_{\text {hv, warning. Therewith, the amount of load }}$ shedding in this scenario cannot be compared to the others.

\section{Conclusion and outlook}

In this paper, an agent-based identification and control against voltage collapse has been presented. The approach extends previously published solutions by combining a robust algorithm for voltage instability detection and a closed-loop scheme for emergency control in a MAS. The latter enables an early and reliable identification of an evolving voltage instability as well as a coordinated activation of countermeasures. As a main feature, the distributed agents only rely on local measurements and limited inter-agent communication making the system real-time capable, adaptive to changing network conditions and robust with respect to communication failures. The performance of the approach has been demonstrated in a co-simulation environment interfacing the MAS with a time-domain simulation of the Nordic Test System.

In future work, the simulation framework of the MAS and the power system, as outlined in Section 3, will be extended by a dedicated simulator for ICT allowing for an explicit analysis of the inter-agent communication, as presented in [28], including an investigation of maximum communication delays. Further, the performance of the MAS will be investigated in a combined transmissiondistribution grid with a detailed representation of underlying active distribution networks characterised by power electronic-interfaced loads and distribution network (voltage) control schemes. Finally, 
This article has been accepted for publication in a future issue of this journal, but has not been fully edited. Content may change prior to final publication in an issue of the journal. To cite the paper please use the doi provided on the Digital Library page.

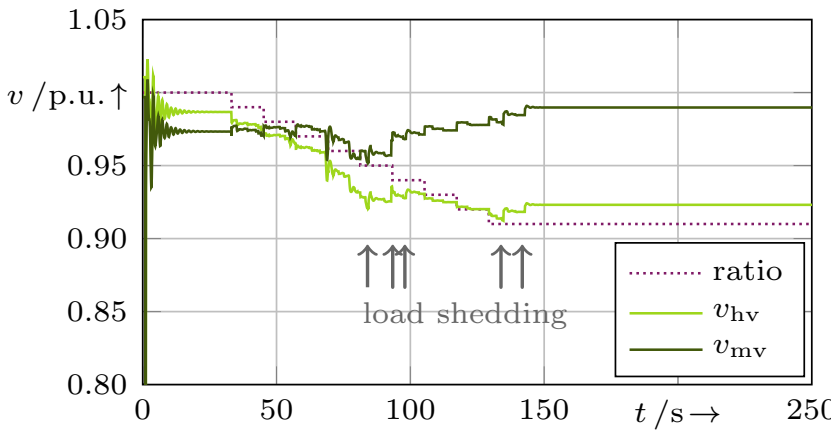

$a$

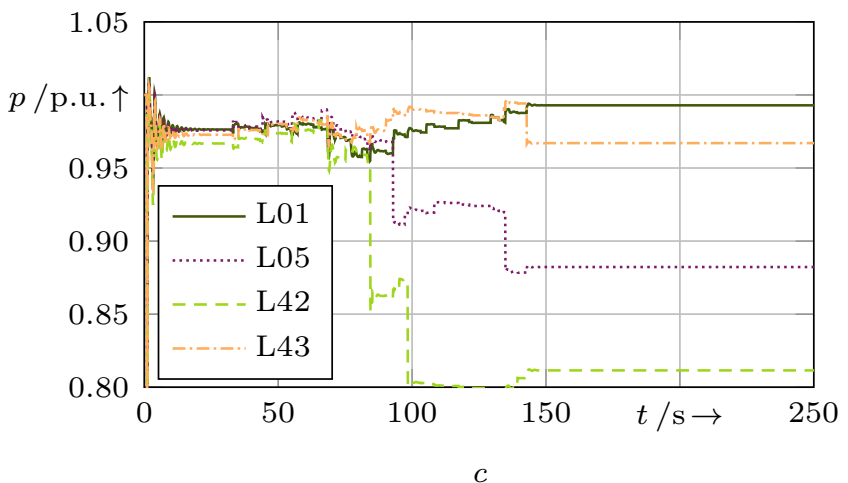

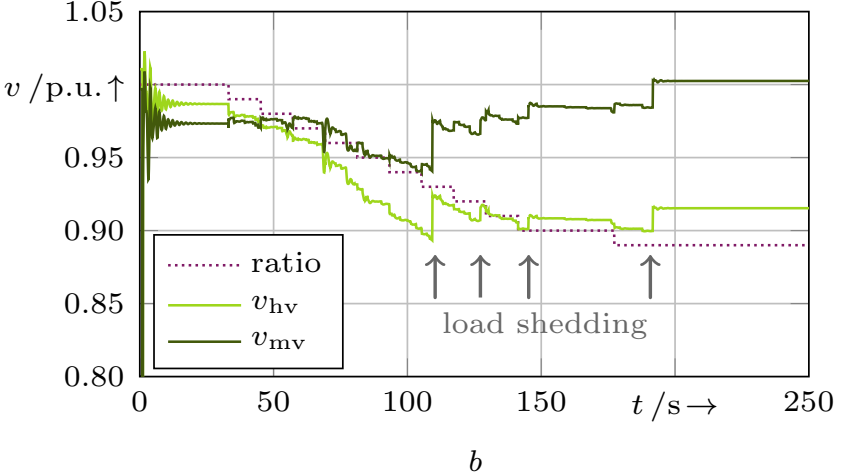

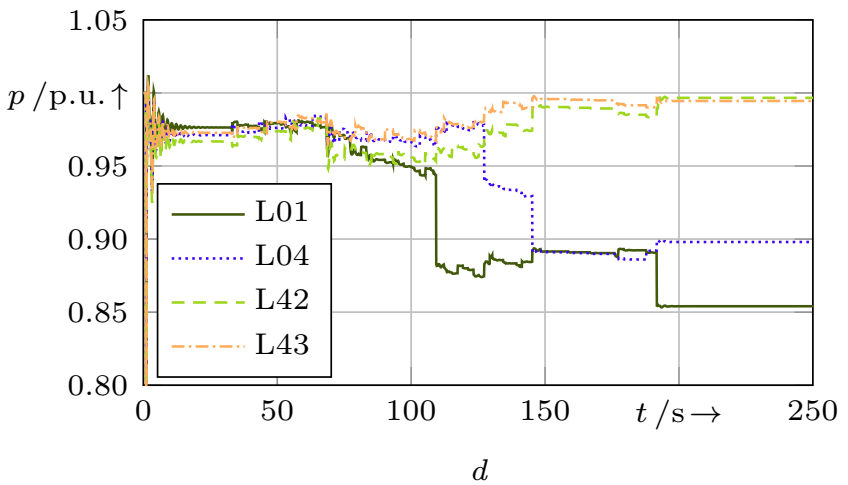

Fig. 7: Evolution of transmission $\left(v_{\mathrm{hv}}\right)$, distribution voltages $\left(v_{\mathrm{mv}}\right)$ and ratio of transformer 1041-01 as well as active power consumption of relevant loads in Scenario D.1 and D.3

a Scenario D.1 - Voltages and tap ratio

$b$ Scenario D.3 - Voltages and tap ratio

$c$ Scenario D.1 - Power consumption

$d$ Scenario D.3 - Power consumption

the proposed agent-based approach will be implemented on a hardware platform with each agent being represented by a single entity. Interfacing the agents with a real-time simulation of the power system enables a hardware-in-the-loop-simulation in order to test and validate the agents' performance in real-life conditions. A first attempt is demonstrated in [29] where agents for autonomous control of power flows were implemented on standard computer hardware which indicates the general economic feasibility.

\section{Acknowledgement}

This work was supported by the German Research Foundation $D F G$ as part of research unit FOR1511: Protection and Control Systems for Reliable and Secure Operation of Electrical Transmission Systems.

Table 5 Results of assessment of voltage measurements and amount of load shedding in Scenarios D. 1 to D.3 (* indicate that alarm is not raised due to outage of communication path)

\begin{tabular}{|c|c|c|c|c|c|c|c|c|c|c|c|c|}
\hline \multirow[b]{2}{*}{ bus } & \multicolumn{4}{|c|}{ D. 1} & \multicolumn{4}{|c|}{ D.2 } & \multicolumn{4}{|c|}{ D.3 } \\
\hline & $\begin{array}{c}\text { time of } \\
\text { warning }\end{array}$ & $\begin{array}{l}\text { time of } \\
\text { alarm }\end{array}$ & $\Delta P_{\mathrm{LS}}$ & $v_{\mathrm{hv}, \mathrm{s}}$ & $\begin{array}{c}\text { time of } \\
\text { warning }\end{array}$ & $\begin{array}{l}\text { time of } \\
\text { alarm }\end{array}$ & $\Delta P_{\mathrm{LS}}$ & $v_{\mathrm{hv}, \mathrm{s}}$ & $\begin{array}{c}\text { time of } \\
\text { warning }\end{array}$ & $\begin{array}{l}\text { time of } \\
\text { hv alarm }\end{array}$ & $\Delta P_{\mathrm{LS}}$ & $v_{\mathrm{hv}, \mathrm{s}}$ \\
\hline 1041 & 55.5 & $-^{*}$ & - & 0.923 & 55.5 & 77.9 & 100 & 0.977 & 55.5 & 109.3 & 50 & 0.915 \\
\hline 1043 & - & - & - & 0.944 & - & - & - & 0.983 & 105.8 & - & - & 0.927 \\
\hline 1044 & 77.9 & - & - & 0.932 & 77.9 & - & - & 0.941 & 77.9 & 127.2 & 50 & 0.906 \\
\hline 1045 & 77.9 & 77.9 & 50 & 0.949 & 77.9 & 77.9 & - & 0.957 & 77.9 & - & - & 0.921 \\
\hline 1022 & - & - & - & 1.019 & - & - & - & 1.013 & 94.3 & - & - & 1.001 \\
\hline 4042 & 72.9 & 76.4 & 50 & 0.963 & 72.9 & $-^{*}$ & - & 0.957 & 72.9 & - & - & 0.921 \\
\hline 4043 & 76.4 & 76.4 & 25 & 0.970 & 76.4 & 76.4 & 25 & 0.970 & 76.4 & - & - & 0.939 \\
\hline 4046 & 80.4 & 80.4 & - & 0.977 & - & - & - & 0.977 & 80.4 & - & - & 0.951 \\
\hline
\end{tabular}


This article has been accepted for publication in a future issue of this journal, but has not been fully edited.

Content may change prior to final publication in an issue of the journal. To cite the paper please use the doi provided on the Digital Library page.

\section{References}

[1] European Network of Transmission System Operators for Electricity (ENTSO-E), 'Network Code on Emergency and Restoration,' March 2015.

[2] CIGRE WG B5.14, 'Wide Area Protection and Control Technologies,' Technical Brochure 644, Sept 2016.

[3] V. Ajjarapu and C. Christy, 'The continuation power flow: a tool for steady state voltage stability analysis', IEEE Trans. Power Syst., vol.7, no.1, pp.416-423, Feb 1992.

[4] T. Zabaiou, L.A. Dessaint, and I. Kamwa, 'Preventive control approach for voltage stability improvement using voltage stability constrained optimal power flow based on static line voltage stability indices,' IET Generation, Transmission Distribution, vol.8, no.5, pp.924-934, May 2014.

[5] M. Glavic and T. Van Cutsem, 'Wide-Area Detection of Voltage Instability From Synchronized Phasor Measurements. Part I: Principle,' IEEE Trans. Power Syst., vol.24, no.3, pp.1408-1416, Aug 2009.

[6] A.G. Beccuti, T.H. Demiray, G. Andersson, and M. Morari, 'A Lagrangian Decomposition Algorithm for Optimal Emergency Voltage Control,' IEEE Trans. Power Syst., vol.25, no.4, pp.1769-1779, Nov 2010.

[7] B. Milosevic and M. Begovic, 'Voltage-stability protection and control using a wide-area network of phasor measurements,' IEEE Trans. Power Syst., vol.18, no.1, pp.121-127, Feb 2003.

[8] K. Vu, M.M. Begovic, D. Novosel, and M.M. Saha, 'Use of local measurements to estimate voltage stability margin,' IEEE Trans. Power Syst., vol.14, no.3, pp.1029-1035, Aug 1999.

[9] W. Li, Y. Wang, and T. Chen, 'Investigation on the Thevenin equivalent parameters for online estimation of maximum power transfer limits,' IET Generation, Transmission, Distribution, vol.4, no.10, pp.1180-1187, Oct 2010.

[10] M. Moradzadeh, R. Boel, and L. Vandevelde, 'Voltage Coordination in Multi-Area Power Systems via Distributed Model Predictive Control,' IEEE Trans. Power Syst., vol.28, no.1, pp.513-521, Feb 2013.

[11] J. Modarresi, E. Gholipour, and A. Khodabakhshian, 'A comprehensive review of the voltage stability indices,' Renewable and Sustainable Energy Reviews, vol.63, pp.1-12, Sept 2016.

[12] M. Glavic and T. Van Cutsem, 'A short survey of methods for voltage instability detection,' in 2011 IEEE PES General Meeting, July 2011, pp.1-8.

[13] S. McArthur, E. Davidson, V. Catterson et al., 'Multi-Agent Systems for Power Engineering Applications - Part I: Concepts, Approaches, and Technical Challenges,' IEEE Trans. Power Syst., vol.22, no.4, pp.1743-1752, Nov 2007.

[14] W. Zhang, W. Liu, X. Wang, L. Liu, and F. Ferrese, 'Distributed Multiple Agent System Based Online Optimal Reactive Power Control for Smart Grids,' IEEE Trans. Smart Grid, vol.5, no.5, pp.2421-2431, Sept 2014.

[15] S.R. Islam, K.M. Muttaqi, and D. Sutanto, 'Multi-agent receding horizon control with neighbour-to-neighbour communication for prevention of voltage collapse in a multi-area power system,' IET Generation, Transmission Distribution, vol.8, no.9, pp.1604-1615, Sept 2014.

[16] F. Baalbergen, M. Gibescu, and L. van der Sluis, 'Agent-based real-time voltage instability detection with maximum loadability index,' in 45th International Universities Power Engineering Conference, Aug 2010, pp.1-6.

[17] D.A. Panasetsky and N.I. Voropai, 'A multi-agent approach to coordination of different emergency control devices against voltage collapse,' in 2009 IEEE Bucharest PowerTech, June 2009, pp.1-7.

[18] T. Nagata and H. Sasaki, 'A multi-agent approach to power system restoration,' IEEE Trans. Power Syst., vol.17, no.2, pp.457-462, May 2002.

[19] M.S. Rahman, M.A. Mahmud, A.M.T. Oo, H.R. Pota, and M.J. Hossain, 'Agent-based reactive power management of power distribution networks with distributed energy generation,' Energy Conversion and Management, vol.120, pp.120-134, July 2016.

[20] R. Olfati-Saber, J.A. Fax, and R.M. Murray, 'Consensus and cooperation in networked multi-agent systems,'Proceedings of the IEEE,vol.95,no.1,pp.215-233, Jan 2007.

[21] L. Robitzky, S. Dalhues, M. Albrecht, S.C. Müller, U. Häger, and C. Rehtanz, 'Agent-Based Prevention of Voltage Collapse in Electrical Transmission Systems,' in 19th Power Systems Computation Conference, June 2016, pp.1-7.

[22] C. Vournas and T. Van Cutsem, 'Local Identification of Voltage Emergency Situations,' IEEE Trans. Power Syst., vol.23, no.3, pp.12391248, Aug 2008.

[23] T. Weckesser, L. Papangelis, C.D. Vournas, and T. Van Cutsem, 'Local identification of voltage instability from load tap changer response,' Sustainable Energy, Grids and Networks, vol.9, pp.95-103, 2017.

[24] M.I. Jordan, 'Notes on recursive least squares,' Oct 1998.

[25] IEEE PES Power System Dynamic Performance Committee, 'Test Systems for Voltage Stability Analysis and Security Assessment,'Technical Report PES-TR19, Aug 2015.

[26] B. Otomega and T. Van Cutsem, 'Undervoltage load shedding using distributed controllers,' IEEE Trans.Power Syst., vol.22, no.4, pp.18981907, Nov 2007

[27] Forum Netztechnik/Netzbetrieb im VDE, 'E-VDE-AR-N-4110: Technical requirements for the connection and operation of customer installations to the medium voltage network,' March 2017.

[28] H. Georg, S.C. Müller, C. Rehtanz, and C. Wietfeld, 'Analyzing Cyber-Physical Energy Systems: The INSPIRE Cosimulation of Power and ICT Systems Using HLA,' IEEE Trans. Ind. Informat., vol.10, no.4, pp.2364-2373, Nov 2014.

[29] N. Dorsch, F. Kurtz, S. Dalhues, L. Robitzky, U. Häger, and C. Wietfeld, 'Intertwined: Software-defined communication networks for multi-agent system-based smart grid control,' in 2016 IEEE International Conference on Smart Grid Communications, Nov 2016, pp.254-259. 